\title{
Open Innovation erfolgreich implementieren
}

Ein großes Potenzial des digitalen Wandels liegt in beschleunigten Innovationszyklen. Nur durch kontinuierliche Innovation sind Unternehmen in der Lage, sich in den volatilen Marktumfeldern permanent neu zu positionieren. Dieses Kapitel beschäftigt sich mit der Fragestellung wie sich diese Innovationen im Unternehmen fördern und besser steuern lassen. Hierbei liegt ein besonderer Fokus auf dem Thema Open Innovation - dem Nutzbarmachen des Innovationspotenzials der Belegschaft oder auch externer Stakeholder durch digitale Technologien. Die Digitalisierung ermöglicht es, dass eine Vielzahl von Akteuren, räumlich und zeitlich unabhängig voneinander an Innovationsprojekten arbeiten. Durch das Öffnen des Innovationsprozesses können im Vergleich zu deutlich kleineren internen Entwicklerteams erhebliche Wettbewerbsvorteile entstehen. Im ersten Beitrag von Allmendinger, Buhleier und Obkircher wird der Frage nachgegangen, ob internes oder externes Crowdsourcing im Unternehmenskontext zu strategischen Vorteilen führt. Hierbei werden die jeweiligen Chancen herausgearbeitet und Handlungsempfehlung für Unternehmen abgeleitet. Der zweite Beitrag von Burchard und Planing beschäftigt sich mit dem verwandten Thema des Crowd-Engineerings und untersucht die Fragestellung wie Personen für die Teilnahme an solchen kollaborativen Projekten motiviert werden können. Hierbei wird insbesondere beleuchtet welche Faktoren dazu führen, dass Ingenieure und Programmierer sich aktiv an Open Innovation Projekten beteiligen.

(C) Springer Fachmedien Wiesbaden GmbH, ein Teil von Springer Nature 2019

A. Lochmahr, P. Müller, P. Planing, T. Popović, Digitalen Wandel gestalten

https.//doi.org/10.1007/978-3-658-24651-8_2.0 